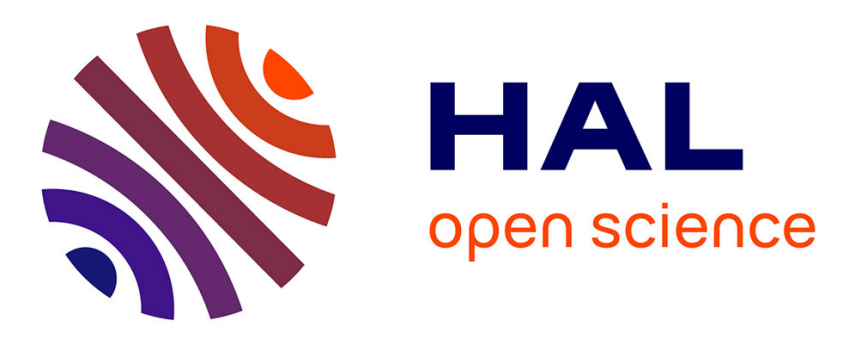

\title{
Flow-level modeling of multi-user beamforming in mobile networks
}

\author{
Ahlem Khlass, Thomas Bonald, Salah Eddine Elayoubi
}

\section{To cite this version:}

Ahlem Khlass, Thomas Bonald, Salah Eddine Elayoubi. Flow-level modeling of multi-user beamforming in mobile networks. RAWNET, May 2014, Hammamet, Tunisia. pp.70 - 77, 10.1109/WIOPT.2014.6850281 . hal-01112973

\section{HAL Id: hal-01112973 https://hal-imt.archives-ouvertes.fr/hal-01112973}

Submitted on 4 Feb 2015

HAL is a multi-disciplinary open access archive for the deposit and dissemination of scientific research documents, whether they are published or not. The documents may come from teaching and research institutions in France or abroad, or from public or private research centers.
L'archive ouverte pluridisciplinaire HAL, est destinée au dépôt et à la diffusion de documents scientifiques de niveau recherche, publiés ou non, émanant des établissements d'enseignement et de recherche français ou étrangers, des laboratoires publics ou privés. 


\section{Flow-level Modeling of Multi-User Beamforming in Mobile Networks}

\author{
Ahlem Khlass \\ Orange Labs \& Telecom ParisTech \\ France \\ ahlem.khlass@orange.com
}

\author{
Thomas Bonald \\ Telecom ParisTech \\ Paris, France \\ thomas.bonald@telecom-paristech.fr
}

\author{
Salah Eddine Elayoubi \\ Orange Labs \\ Issy-Les-Moulineaux, France \\ salaheddine.elayoubi@orange.com
}

\begin{abstract}
Among the several features that are foreseen for increasing the capacity of cellular systems, Multi-user multipleinput multiple-output (MU-MIMO) is considered as a key enabling technology. Particularly, MU-beamforming is a powerful means of increasing the system capacity and throughput by creating several spatial signals to different users on the same time/frequency resource. In this paper, we develop an analytical model for MU-beamforming based on queuing theory combined with network simulations. The proposed framework is used for evaluating the potential gains in terms of capacity and throughput in LTE-Advanced system. Several scenarios are studied, with and without beamforming. Results show an important gain of SU-beamforming over the classical system without beamforming and a further gain of MU-beamforming, especially at high loads.

Index Terms- Multi-user beamforming, LTE-Advanced, Flow Level Modeling, Queuing Theory, Simulations.
\end{abstract}

\section{INTRODUCTION}

Nowadays, the wireless networks industry is moving toward LTE system which is expected to improve the performance in terms of spectral efficiency and user throughput. In its release 8 , it enables to achieve $300 \mathrm{Mb} / \mathrm{s}$ for the downlink and 75 $\mathrm{Mb} / \mathrm{s}$ for the uplink with the utilization of OFDM and single user-MIMO (SU-MIMO) transmission. Then, in order to meet the requirements of the IMT-Advanced systems, LTE has been further enhanced to LTE-Advanced which is able to achieve a target throughput of $1 \mathrm{~Gb} / \mathrm{s}$ on the downlink and $500 \mathrm{Mb} / \mathrm{s}$ on the uplink with advanced physical layer techniques [1] [2] including carrier aggregation [3], enhanced multi-antenna schemes and Coordinated Multi-Point transmission technique (CoMP) [4]. Particularly, multi-user MIMO (MU-MIMO) is considered as a key enabling technology for LTE-Advanced that enables to achieve the expected performance [5].

Indeed, in downlink MU-MIMO system, the Base Station (BS) creates different spatial signals to multiple Mobile Stations (MS) on the same time/frequency resource. Accordingly, the scheduling opportunities for each MS is largely improved compared to a SU-MIMO system where only a single user exploits the spatial gain offered by multi-antenna systems [6]. However, this operation introduces interference between co-scheduled users within the same cell in addition to the interference from adjacent cells. Therefore, advanced precoding/beamforming techniques and a well designed scheduling scheme need to be implemented in order to combat this intra-cell interference and increase the system capacity.
While a large number of papers deals with the physical layer aspects of MU-MIMO by investigating the optimal schemes with low complexity, we propose in this paper a flow level modeling of MU-MIMO in different scenarios. Indeed, existing performance evaluations of MU-MIMO are based on system-level simulations with a static number of users in different cells (e.g. [7] [8] [9]); they do not capture the dynamics of users' behavior in the system. Moreover, they do not reflect the actual distribution of users in the cell since those at cell edge stay longer in the cell for finishing their service due to a low data rate [10]. However, in this work, we propose an analytical method based on queuing theory that captures the dynamics of arrivals and departure processes. It is practically interesting and, to the best of our knowledge, has not been proposed yet.

In this paper, we consider a special MIMO scheme which is transmit beamforming that permits to concentrate the energy towards one or more directions. Particularly, we evaluate the expected gains of MU-beamforming in terms of throughput and capacity in different scenarios. We start with SUbeamforming system, then we investigate the MU-scheduling impact under different network configurations. We propose for each scenario a flow-level model and we evaluate its performance using both Markovian analysis and network simulations in different load conditions. Results show an important gain of SU-beamforming over the classical system without beamforming and a further gain of MU-beamforming, especially at high loads.

The remainder of this paper is organized as follows. Section II presents a basic background of MIMO system and MUMIMO framework. Section III describes the model used in this paper. We present in section IV the flow-level analysis for the SU-beamforming and the MU-beamforming in the presence of three and four serving beams. Section $\mathrm{V}$ gives the results using network simulations. Section VI concludes the paper.

\section{BACKGROUND}

\section{A. MIMO System}

One of the key technique of advanced communication systems is the MIMO transmission which enables multiple antennas at both receiver and transmitter, with additional processing, to improve the system performance in terms of 
coverage, capacity and robustness. There are basically three main advantages from MIMO transmission [11]:

- Diversity gain: by using the spatial diversity provided by the multiple antennas in order to improve the system robustness and reliability.

- Spatial multiplexing gain: by transmitting multiple data streams to a single or multiple users on multiple spatial dimensions (i.e. layers) in order to improve the user's spectral efficiency.

- Array gain: by concentrating the energy in one or more directions via precoding/beamforming functionality, thus increasing the data rates.

Various scenarios are proposed for LTE downlink; they are referred as Transmission Modes (TM) in releases' specification. The final MIMO scheme depends on the network deployment and the propagation conditions of the different users.

\section{B. MU-MIMO Framework}

The MU-MIMO framework has been introduced for LTEAdvanced standard and considered as a key enabler for achieving high spectral efficiency. It enables a BS to co-schedule a set of MSs on the same time/frequency resource. However, this operation introduces additional intra-cell interference. Therefore, advanced precoding and scheduling methods need to be implemented to get full benefit of MU-MIMO transmission.

1) MU-Precoding: The key issue with the MU-precoding is to find a compromise between performance gain, Channel State Information (CSI) requirement and design complexity. The Dirty Paper Coding (DPC) [12] enables to achieve an optimal capacity but is impractical in real systems due to its complexity and full CSI requirement. Alternatively, linear precoding methods, such as zero-forcing (ZF) [13], appear to be more robust to erroneous CSI and with a low complexity relatively to other methods.

2) User selection/scheduling: Different factors affect the MU-scheduler performance including the wireless environment since a minimum separation between users is required. This is only possible through narrow antenna beam patterns. Also, the cell load is an important factor; indeed highly loaded cells provide more user grouping possibilities than lightly loaded cells where it is more efficient to schedule a single user. Different schedulers have been proposed in literature for MU-MIMO (e.g. [14] and reference therein).

Since we consider a beamforming scheme, the MU scheduling can be performed using:

- Max-Sum scheduler. The aim is to find the optimal set of users covered by different beams which maximizes a total utility function.

- Max-iterate scheduler. A first user is chosen according to a certain metric, then users from adjacent beams are blocked and the same process is repeated until reaching the maximum number of active beams or that there are no more users to schedule.

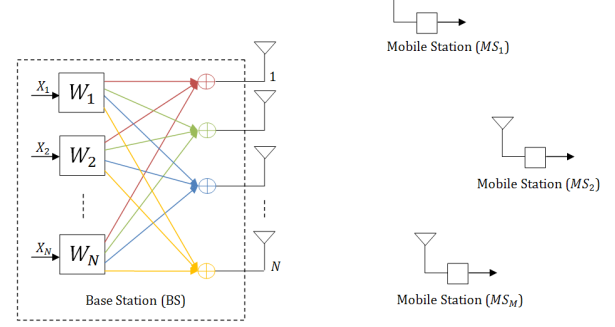

Fig. 1. Block diagram of the MU-beamforming system.

\section{MODEL}

\section{A. Physical layer aspects}

We consider a downlink multi-user environment consisting on a base station (BS) with $N$ transmit antennas communicating with $M$ mobile stations (MS). We focus on the beamforming system represented by the block diagram shown in figure 1 where the data information blocks are mapped into layers, then to the antenna ports by means of the precoding/beamforming functionality. We investigate two possible schemes [15]: the one-layer beamforming where a single layer is assigned to one MS (i.e. SU-beamforming) and dual-layer beamforming where the two layers are assigned to two separte MSs (i.e. MU-beamforming).

Let $X_{i}$ and $W_{i}$ denote the transmitted data intended to user $i$ and its transmit beamforming vector. When one-layer beamforming is used, the received signal at $M S_{i}$ is given by:

$$
Y_{i}=H_{i} W_{i} X_{i}+I_{i}+N_{i},
$$

where $H_{i}, I_{i}$ and $N_{i}$ denote the channel gain from the BS to the $i$ th MS, the inter-cell interference and an Additive White Gaussian Noise (AWGN) vector at $M S_{i}$, respectively. The corresponding SINR is given by:

$$
S I N R_{i}=\frac{\left\|H_{i} W_{i}\right\|^{2}}{\left\|I_{i}\right\|^{2}+\left\|N_{i}\right\|^{2}} .
$$

However, when dual-layer beamforming is deployed, the received signal at $M S_{i}$ is expressed by:

$$
Y_{i}=H_{i} W_{i} X_{i}+H_{i} \sum_{\substack{l=1 \\ l \neq i}}^{2} W_{l} X_{l}+I_{i}+N_{i}
$$

In Equation 1, the four terms represent the desired signal, the multi-user interference, the inter-cell interference and the noise, respectively. Hence, the SINR at user $i$ is:

$$
\operatorname{SIN}_{i}=\frac{\left\|H_{i} W_{i}\right\|^{2}}{\left\|H_{i} \sum_{\substack{l=1 \\ l \neq i}}^{2} W_{l}\right\|^{2}+\left\|I_{i}\right\|^{2}+\left\|N_{i}\right\|^{2}} .
$$

Using the Shannon's formula, we can express the transmission rate by:

$$
R_{i}=W \log \left(1+S I N R_{i}\right),
$$

where $W$ denotes the system bandwidth. 


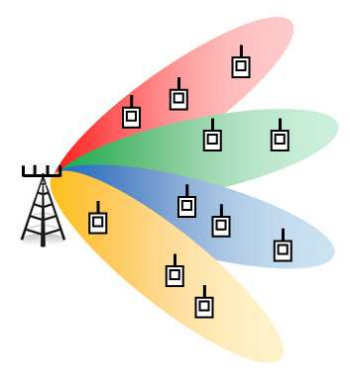

Fig. 2. System model.

Note that, in this paper, we do not address the calculation of the optimal beamforming weights as done in several studies (e.g. [9] and references therein). We assume that the BS has an arbitrary number of transmit antennas that can provide $J$ spaced beams per sector as shown in figure 2 . The $J$ predefined beams are considered as a fixed codebook either in SU or MU-beamforming modes. This model is used in the subsequent analysis.

\section{B. Scheduling aspects}

We consider a centralized scheduler per radio site which decides at each time slot the set of users to co-schedule on the same time/frequency resource. Different schedulers can be performed as seen in section II-B2 that aim at getting full benefit from multi-user diversity and so improving the system capacity while minimizing the intra-cell interference.

In this work, we consider a Max-Iterate scheduler based on Round Robin which selects a first user at random, blocks all users in the adjacent beams and iterates until reaching the maximum number of active beams or that there are no more users to schedule. The considered MU-scheduling is described in algorithm 1.

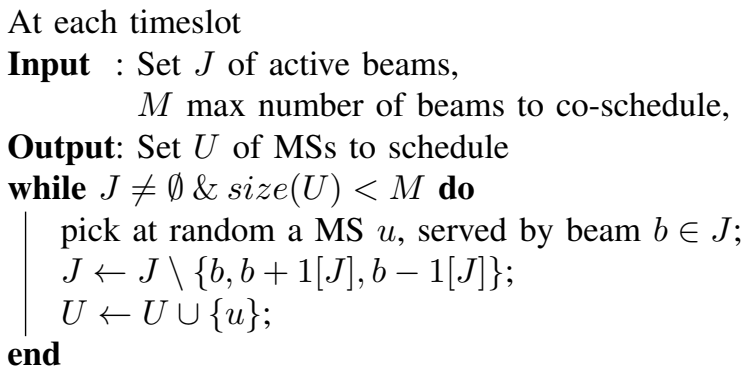

Algorithm 1: MU-Scheduling algorithm.

Although this scheduler is simple and does not take into account the radio channel conditions, it remains a good approximation of any opportunistic scheduler like proportional fair when the channel variations are limited or too fast to be exploited.

\section{Traffic aspects}

We assume elastic traffic. Data flows arrive according to a Poisson process of intensity $\lambda$ in the reference cell. Each flow stays in the system as long as the corresponding data have not been successfully transmitted to the MS. Note that the users are assumed to be static during the whole flow duration. Flow sizes are assumed to be independent and exponentially distributed with mean $\sigma$ bits. The traffic intensity $A$ in the reference cell is the product $\lambda \times \sigma$ in bit/s.

\section{Flow-LeVel Analysis}

We start with a SU-beamforming system in the presence of $J$ serving beams, then we investigate the impact of MUscheduling under different network configurations.

We focus on a given sector. We consider a set $C$ of classes of MSs indexed by $i$ to reflect the different radio conditions in the reference sector. Classes are grouped into subsets $C_{j}$ for $j \in \mathcal{J}=\{1, \ldots, J\}$ in the presence of $J$ serving beams. Since class- $i$ flows arrive at rate $\lambda_{i}$, the corresponding load is:

$$
\rho_{i}=\frac{\lambda_{i} \sigma}{R_{i}}
$$

where $R_{i}$ is the transmission rate at class- $i$ expressed in expression (2).

\section{A. SU-beamforming}

According to SU-beamforming system, the scheduler selects at random a user covered by a particular beam. This latter is served alone and blocks all other users in the sector. Let $Z_{j}(t)$ be the total number of users in beam $B_{j}$ at time $t$, for $j \in \mathcal{J}$. Denote by $\vec{Z}(t)$ the corresponding vector. Whenever $\vec{Z}(t)=\vec{z}$, the scheduler selects a user in $B_{j}$ a fraction of time of [16]:

$$
\phi_{j}(\vec{z})=\frac{z_{j}}{\bar{z}},
$$

where $\bar{z}$ denotes the total number of users in whole sector. The queuing system is described by the following transition rates of the Markov process $\vec{Z}(t)$ from state $\vec{z}$ to state $\vec{z}^{\prime}$ :

$$
q\left(\vec{z}, \vec{z}^{\prime}\right)= \begin{cases}\bar{\lambda}_{j} & \text { for } \vec{z}^{\prime}=\vec{z}+\vec{e}_{j} j \in \mathcal{J} \\ \phi_{j}(\vec{z}) \frac{\bar{R}_{j}}{\sigma} & \text { for } \vec{z}^{\prime}=\vec{z}-\vec{e}_{j}, j \in \mathcal{J}\end{cases}
$$

where $\vec{e}_{j}$ is the unit vector on component $j$, for $j \in \mathcal{J}$.

The system corresponds to a multi-class processor sharing queue with total load $\rho=\sum_{j \in \mathcal{J}} \bar{\rho}_{j}$ where

$$
\bar{\rho}_{j}=\frac{\bar{\lambda}_{j} \sigma}{\bar{R}_{j}}
$$

The arrival rate per beam $\bar{\lambda}_{j}$ is given by

$$
\bar{\lambda}_{j}=\sum_{i \in C_{j}} \lambda_{i}
$$

and the mean transmission rate

$$
\bar{R}_{j}=\frac{\sum_{i \in C_{j}} p_{i}}{\sum_{i \in C_{j}} \frac{p_{i}}{R_{i}}}
$$

with

$$
\sum_{i \in C} p_{i}=\sum_{i \in C} \lambda_{i} / \lambda=1
$$


In order to investigate the performance of such system, we propose lemma (1).

Lemma 1. Considering a Whittle network [17] of J queues of load $\rho_{j}$, the stationary distribution of the process $z(t)$ is:

$$
\pi(z)=\pi(0) \Phi(z) \prod_{j=1}^{J} \rho_{j}^{z_{j}},
$$

where $\Phi(z)$ denotes the inverse of the product of service capacities along any direct path from state $z$ to state 0 . Let

$$
G(\rho)=\sum_{z} \Phi(z) \prod_{j=1}^{J} \rho_{j}^{z_{j}} .
$$

For each queue j, we have:

- the mean number of users in each queue $j$

$$
E\left(z_{j}\right)=\rho_{j} \frac{\partial G(\rho)}{\partial \rho_{j}} \frac{1}{G(\rho)} .
$$

- the flow-throughput

$$
\gamma_{j}=R_{j} \frac{\partial}{\partial \rho_{j}} \ln (G(\rho)) .
$$

Proof. Based on the normalization condition where $\sum_{z} \pi(z)=1$, we have:

$$
\pi(0)=\frac{1}{G(\rho)},
$$

Then, the mean number of users in each queue is given by:

$$
\begin{aligned}
E\left(z_{j}\right) & =\sum_{\substack{z \\
z_{j} \neq 0}} z_{j} \pi(0) \Phi(z) \prod_{j=1}^{J} \rho_{j}^{z_{j}} \\
& =\rho_{j} \frac{\partial G(\rho)}{\partial \rho_{j}} \frac{1}{G(\rho)} .
\end{aligned}
$$

The mean sojourn time in each queue follows from Little's law [18] :

$$
\tau_{j}=\frac{E\left(z_{j}\right)}{\lambda_{j}} .
$$

Hence, we obtain the flow throughput:

$$
\begin{aligned}
\gamma_{j}=\frac{\sigma}{\tau_{j}} & =R_{j} \frac{\rho_{j}}{E\left(z_{j}\right)} \\
& =R_{j} \frac{\partial}{\partial \rho_{j}} \ln (G(\rho)) .
\end{aligned}
$$

The multi-class processor sharing queue can be viewed as a Whittle network [17]. Under the stability condition $\rho<1$, the stationary distribution of the system describing the number of flows in each beam of the reference cell is given by:

$$
\pi(\vec{z})=\pi(\overrightarrow{0}) \frac{\bar{z} !}{\prod_{j} z_{j} !} \prod_{j} \rho_{j}^{z_{j}},
$$

Then, using lemma (1), we obtain the mean number of active users in beam- $j$ flows:

$$
E\left(z_{j}\right)=\frac{\bar{\rho}_{j}}{1-\rho} .
$$

We deduce the flow throughput in beam $B_{j}$

$$
\gamma_{j}=\bar{R}_{j}(1-\rho)
$$

The mean throughput in the cell is given by:

$$
\gamma=R(1-\rho)
$$

where $R$ refers to the total cell capacity defined as the maximum traffic intensity that can be handled.

We note that the flow throughput decreases linearly with the load from the maximum data rate $\bar{R}_{j}$ when $\rho=0$ to 0 when the reference cell is fully loaded $(\rho=1)$. However, in real cases, this is not a linear decrease since the transmission rates are not fixed and depend on the load of neighboring cells and the caused interference.

\section{B. MU-beamforming}

To gain insight into the MU gain, we consider the two-layer beamforming system in the presence of $J=3$ and $J=4$ serving beams per sector.

1) MU-beamforming in the presence of 3 beams: According to the MU-scheduling algorithm aforementioned, the system switches between a SU and MU-beamforming system depending on the first selected user. In fact, if a user covered by the beam $B_{1}$ is selected first, then only those covered by $B_{3}$ are eligible to be selected next. However, when a user in beam $B_{2}$ is selected first, all others covered by adjacent beams namely $B_{1}$ and $B_{3}$ are blocked.

Hence, users in beams $B_{1}$ and $B_{3}$ are selected a fraction of time of:

$$
\phi_{1}(\vec{z})=\phi_{3}(\vec{z})=\frac{z_{1}+z_{3}}{\bar{z}}
$$

and those in beam $B_{2}$ a fraction of time:

$$
\phi_{2}(\vec{z})=\frac{z_{2}}{\bar{z}} .
$$

The system corresponds to a set of three coupled processorsharing queues with state-dependent service rates. The queuing system is described by the transition rates given in (3) with $J=3$. This Markov process is reversible and equivalently, the queuing system corresponds to a Whittle network. The stationary distribution of the Markov process $\vec{Z}(t)$ is then given by:

$$
\begin{aligned}
\pi(\vec{z}) & =\frac{\left(1-\bar{\rho}_{1}-\bar{\rho}_{2}\right)\left(1-\bar{\rho}_{3}-\bar{\rho}_{2}\right)}{\left(1-\bar{\rho}_{2}\right)} \\
& \times\left(\begin{array}{c}
\bar{z} \\
z_{1}+z_{3}
\end{array}\right) \bar{\rho}_{1}^{z_{1}} \bar{\rho}_{2}^{z_{2}} \bar{\rho}_{3}^{z_{3}},
\end{aligned}
$$

under the stability condition that:

$$
\bar{\rho}_{1}+\bar{\rho}_{2}<1, \quad \bar{\rho}_{3}+\bar{\rho}_{2}<1 .
$$




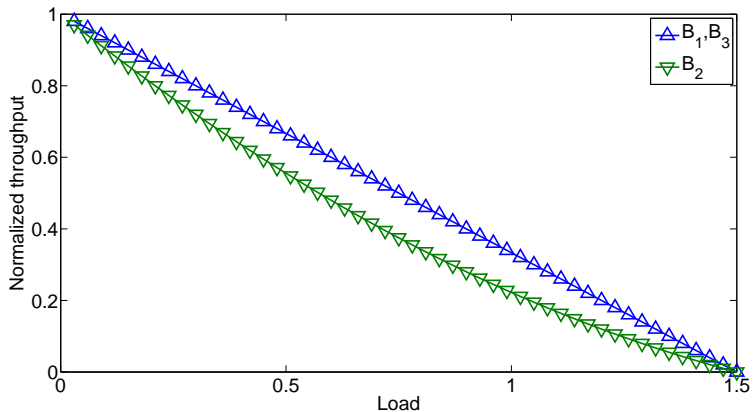

(a) per beam

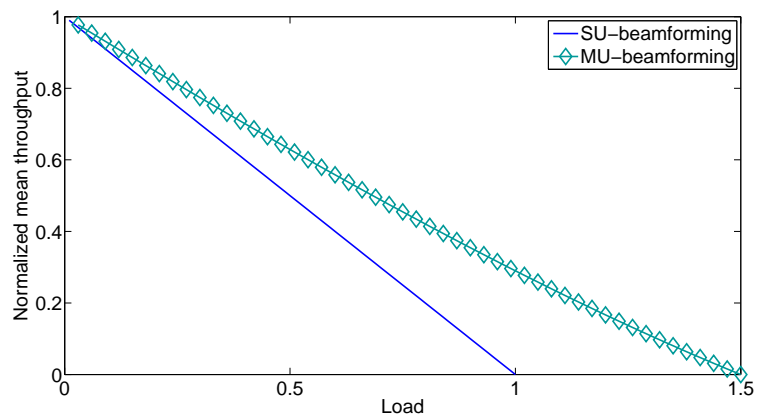

(b) in average

Fig. 3. Normalized user throughput with MU-beamforming with 3 beams.

We use lemma (1), we deduce from equation (5) the mean number of flows in each beam

$$
\begin{gathered}
E\left(z_{1}\right)=\frac{\bar{\rho}_{1}}{1-\bar{\rho}_{1}-\bar{\rho}_{2}}, \\
E\left(z_{2}\right)=\frac{\bar{\rho}_{2}}{1-\bar{\rho}_{2}}\left(1+\frac{\bar{\rho}_{1}}{1-\bar{\rho}_{1}-\bar{\rho}_{2}}+\frac{\bar{\rho}_{3}}{1-\bar{\rho}_{3}-\bar{\rho}_{2}}\right) . \\
E\left(z_{3}\right)=\frac{\bar{\rho}_{3}}{1-\bar{\rho}_{3}-\bar{\rho}_{2}},
\end{gathered}
$$

and from equation (6) the flow throughput in each beam:

$$
\begin{gathered}
\bar{\gamma}_{1}=\bar{R}_{1}\left(1-\bar{\rho}_{1}-\bar{\rho}_{2}\right), \quad \bar{\gamma}_{3}=\bar{R}_{3}\left(1-\bar{\rho}_{3}-\bar{\rho}_{2}\right), \\
\bar{\gamma}_{2}=\frac{\bar{R}_{2}\left(1-\bar{\rho}_{2}\right)}{1+\frac{\bar{\rho}_{1}}{1-\bar{\rho}_{1}-\bar{\rho}_{2}}+\frac{\bar{\rho}_{3}}{1-\bar{\rho}_{3}-\bar{\rho}_{2}}} .
\end{gathered}
$$

Figure 3 shows the results in terms of normalized throughput per beam and in average compared to that obtained with SU-beamforming. We see that the capacity region is improved by one half when using MU-beamforming since the system with 3 beams switches between SU and MU. In fact, depending on the first selected user, either the two beams at extremities are active or the one at the middle.

2) MU-beamforming in the presence of 4 beams: In this case of $J=4$ beams per sector, the scheduling algorithm is always able to select two users from two different beams. In fact, selecting a user from any beam leaves always the possibility to select another user from another beam (except from the adjacent ones). For instance, if a user covered by beam $B_{2}$ is selected, this blocks those covered by adjacent beams $B_{1}$ and $B_{3}$. Then only users covered by beam $B_{4}$ are eligible to be co-scheduled jointly with the selected user from $B_{2}$. However, when a user covered by beam $B_{1}$ is selected, this gives the possibility to users covered by both $B_{3}$ and $B_{4}$ to be scheduled. We differentiate in this case between two scheduling behaviors:

a) Fixed scheduling scheme: As its name suggests, the scheduling scheme in the cell is predefined since the scheduler can either select a user covered by a beam whose index is even or odd. Indeed, if a user covered by $B_{1}$ is selected, then only a user from beam $B_{3}$ can be co-scheduled. Accordingly, at a given time slot, either beams $B_{1}+B_{3}$ or $B_{2}+B_{4}$ can be active at a given time slot, regardless of their corresponding loads. This can be expressed in terms of fractions of time during which the beams are active:

$$
\begin{aligned}
& \phi_{1}(\vec{z})=\phi_{3}(\vec{z})=\frac{z_{1}+z_{3}}{\bar{z}}, \\
& \phi_{2}(\vec{z})=\phi_{4}(\vec{z})=\frac{z_{2}+z_{4}}{\bar{z}} .
\end{aligned}
$$

The system corresponds in this case to a set of four coupled processor-sharing queues. The queuing system is similar to that described in (3) with $J=4$. The reversibility is maintained and we deduce the stationary distribution of the Markov process $\vec{Z}(t)$ :

$$
\pi(\vec{z})=\pi(\overrightarrow{0}) \times\left(\begin{array}{c}
\bar{z} \\
z_{1}+z_{3}, z_{2}+z_{4}
\end{array}\right) \prod_{j=1}^{j=4} \bar{\rho}_{j}^{z_{j}},
$$

under the stability condition:

$$
\bar{\rho}_{1}+\bar{\rho}_{3}<1, \quad \bar{\rho}_{2}+\bar{\rho}_{4}<1 .
$$

For simplicity, we suppose that all beams have the same load such as

$$
\bar{\rho}_{j}=\bar{\rho}, \forall j \in \mathcal{J} .
$$

We use lemma (1) and we deduce from equation (7):

$$
\pi(0)=\frac{(1-2 \bar{\rho})^{3}}{1-2 \bar{\rho}+(2 \bar{\rho})^{2}} .
$$

Hence, the mean number of users in each beam $B_{j}, \forall j \in \mathcal{J}$, follows from equation (5) so that

$$
E\left(z_{j}\right)=\frac{\bar{\rho}}{(1-2 \bar{\rho})} \frac{1-\bar{\rho}+\bar{\rho}^{2}}{1-2 \bar{\rho}+2 \bar{\rho}^{2}},
$$

and the corresponding flow throughput:

$$
\bar{\gamma}_{j}=\bar{R}_{j}\left(\frac{\bar{\rho}}{(1-2 \bar{\rho})} \frac{1-\bar{\rho}+\bar{\rho}^{2}}{1-2 \bar{\rho}+2 \bar{\rho}^{2}}\right)^{(-1)} .
$$

Note here that $\bar{R}_{j}$ refers to the transmission rate in $B_{j}$ in the presence of another active beam. 
b) Flexible scheduling scheme: Under the flexible scheduling, the load in each beam is taken into account. Indeed, if a user in beam $B_{1}$ is selected, this leaves the possibility to users covered by either beam $B_{3}$ or $B_{4}$ to be co-scheduled depending on their loads. The fractions of time during which beams are active is then given by:

$$
\begin{gathered}
\tilde{\phi}_{1}(\vec{z})=\frac{z_{1}+z_{3}}{\bar{z}}+\frac{z_{4}}{\bar{z}} \frac{z_{1}}{z_{1}+z_{2}}, \\
\tilde{\phi}_{2}(\vec{z})=\frac{z_{2}}{\bar{z}}+\frac{z_{4}}{\bar{z}} \frac{z_{2}}{z_{1}+z_{2}}, \\
\tilde{\phi}_{3}(\vec{z})=\frac{z_{3}}{\bar{z}}+\frac{z_{1}}{\bar{z}} \frac{z_{3}}{z_{3}+z_{4}}, \\
\tilde{\phi}_{4}(\vec{z})=\frac{z_{4}+z_{2}}{\bar{z}}+\frac{z_{1}}{\bar{z}} \frac{z_{4}}{z_{3}+z_{4}} .
\end{gathered}
$$

The system corresponds to a set of four coupled processorsharing queues. The queuing system is the similar to that presented in (3) except that $\phi_{j}(\vec{z})$ is replaced by $\tilde{\phi}_{j}(\vec{z})$ for $j=1, . ., 4$. This Markov process is not reversible. Therefore, the stationary distribution of the number of active flows in the system is not deduced as done previously for the fixed scheduling scheme.

The performance of the fixed and flexible scheduling schemes in a MU-beamforming system with $J=4$ beams is obtained by simulation of $10^{6}$ jumps of the Markov process and plotted in figure 4 in terms of normalized throughput per beam and in average compared to the SU-beamforming. We see that the system capacity is twice that observed with a SU-beamforming. Moreover, we see that the flexible scheme favors the beams at extremities ( $B_{1}$ for instance) where users experience higher performance compared to those in other beams (e.g. $B_{2}$ ) since they have more chances to be selected at the expense of other beams. Thus, in average, the flexible MU-scheduling performs slightly better than the fixed scheme, except at high loads where the two schemes perform similarly.

In summary, we observe an important improvement of the system capacity when increasing the number of users to coschedule and the number of beams as well. For practical issues, we decide to limit the number of beams to $J=4$ per sector and the number of users to co-schedule to two users, but this analysis can be further extended to any MU-beamforming scheme.

\section{NUMERICAL RESULTS}

While the numerical results presented in the previous section were based on normalized rates and aimed at illustrating the derived models, we present in this section a numerical analysis where the analytical models take as input realistic rates from an LTE-advanced simulator.

\section{A. Achievable rates}

We first present network simulations results that are used to calculate the achievable rates is $\mathrm{SU}$ and MU-beamforming in comparison to a classical system without beamforming.

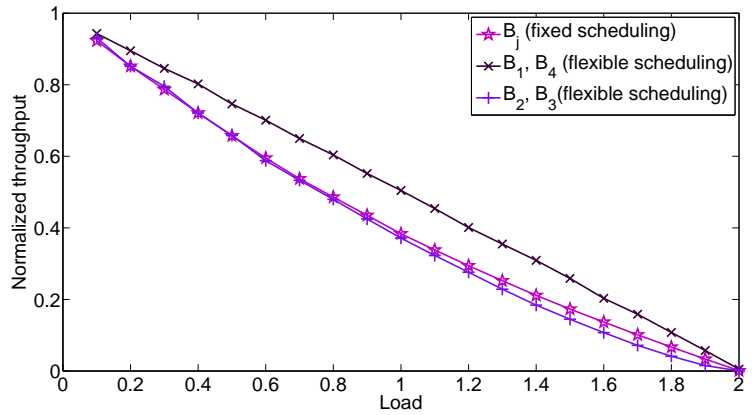

(a) per beam

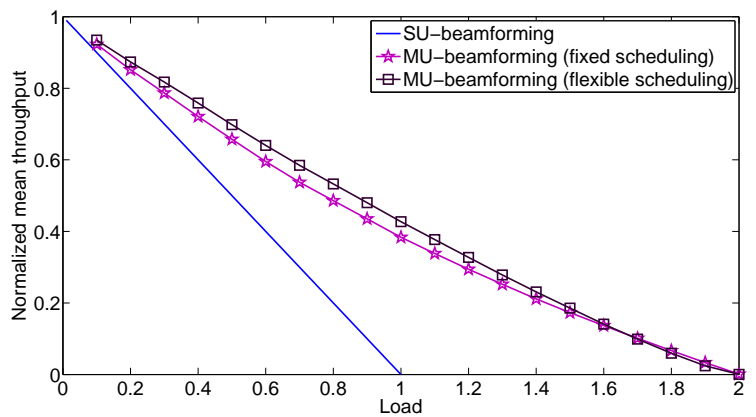

(b) in average

Fig. 4. Normalized user throughput with MU-beamforming with 4 beams.

System level evaluation is conducted to compare the performance of a classical system without beamforming to SUbeamforming and MU-beamforming systems in LTE-advanced network settings.

Considering the system parameters summarized in table I, we illustrate in figure 5 , the transmission rates at different positions of the reference site on the basis of 100 independent network states. The average values are given in table II in different scenarios. Since our purpose is to evaluate the MU gain of MU-beamforming, we do not consider advanced features of LTE-advanced networks like OFDMA for instance. We assume also that the base station transmit at full power using the whole frequency band.

We see at first that SU-beamforming enables to achieve higher transmission rates compared to a classical system without beamforming. Indeed, concentrating the transmit power on the target MS increases the received power and decreases the interference caused to other MSs. However, with MUbeamforming, the transmission rates are less important due to intra-cell interference caused by the presence of two active beams per cell.

\section{B. Performance results}

We address in this section the performance results when combining the Markovian analysis of section (IV) with the network simulations. For different traffic intensities, we plot the average throughput with MU-beamforming and SUbeamforming in comparison to the classical system without beamforming. Results are shown in figure 6 . 


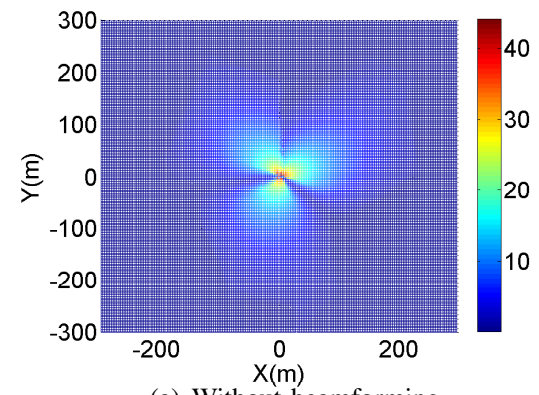

(a) Without beamforming

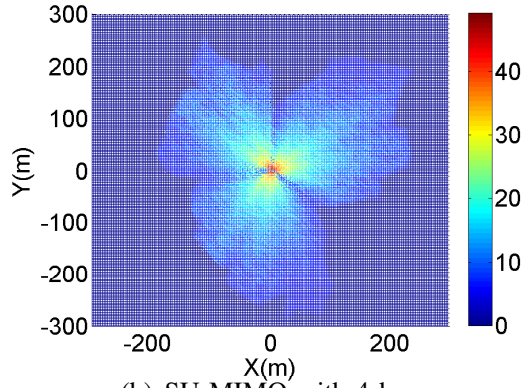

(b) SU-MIMO with 4 beams

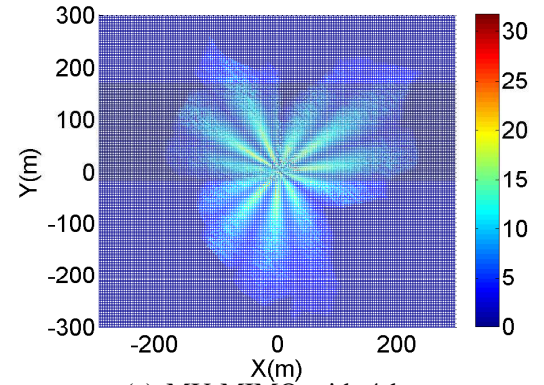

(c) MU-MIMO with 4 beams

Fig. 5. Peak transmission rates in Mbit/s.

TABLE I

SIMULATION PARAMETERS

\begin{tabular}{|l|c|}
\hline Parameter & Value \\
\hline Site layout & $\begin{array}{c}19 \text { sites, } \\
\text { 3 sectors per site }\end{array}$ \\
\hline Inter-site distance & $500 \mathrm{~m}$ \\
\hline Cell radius & $350 \mathrm{~m}$ \\
\hline System bandwidth & $5 \mathrm{MHz}$ \\
\hline Carrier frequency band & $2.6 \mathrm{GHz}$ \\
\hline Antenna model & $3 \mathrm{GPP}$ model [19] \\
\hline Propagation model & Hata model [20] \\
\hline Path loss exponent & 3.5 \\
\hline Load at neighboring sectors & $50 \%$ \\
\hline
\end{tabular}

TABLE II

AVERAGE TRANSMISSION RATES

\begin{tabular}{|c|c|}
\hline System & Rate (Mbit/s) \\
\hline Without beamforming & 4.66 \\
\hline SU-beamforming with 3 beams & 7.55 \\
\hline SU-beamforming with 4 beams & 8.02 \\
\hline MU-beamforming with 3 beams & 5.51 \\
\hline MU-beamforming with 4 beams & 4.95 \\
\hline
\end{tabular}

We notice an important gain of SU-beamforming over the classical system without beamforming and a further gain of MU-beamforming, especially at high loads. Indeed, the system capacity improves considerably by $30 \%$ when performing MUbeamforming with 4 beams under the fixed scheduling scheme and around $20 \%$ with 3 beams since this system switches between SU and MU-beamforming.

Note that these results are valid for a constant load in neighboring cells (50\%). However, in real cases, the flow throughput does not decrease linearly since the transmission rates depend on the interference caused by neighboring cells.

\section{CONCLUSION}

In this paper, we proposed a flow-level modeling of MUbeamforming in cellular networks. We evaluated its performance by combining Markovian analysis with network simulations. We observed that MU-beamforming enables to improve the performance of a SU-beamforming in terms of capacity, especially at high loads. Results were given in a system providing 3 and 4 beams and a maximum of two users

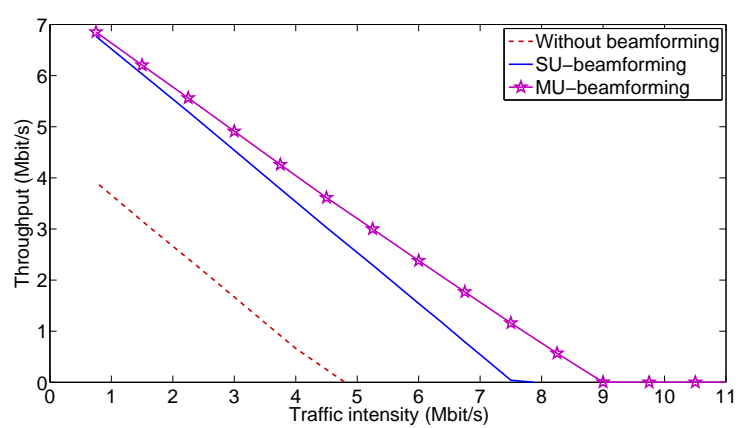

(a) Case with 3 beams

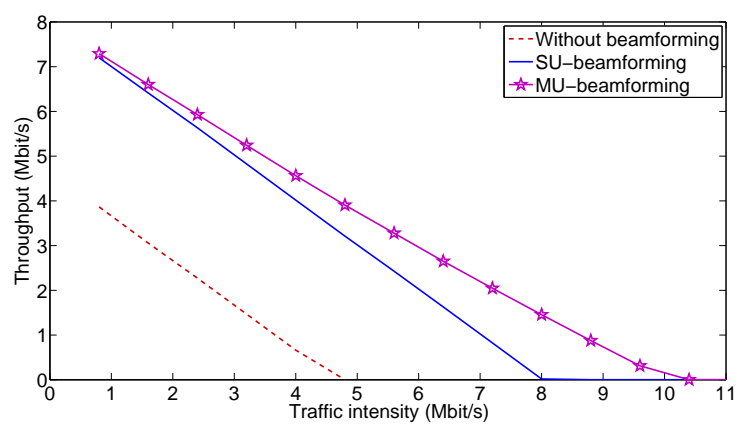

(b) Case with 4 beams

Fig. 6. Mean user throughput vs. traffic intensity.

to co-schedule. Notice that all the Markovian analysis and the simulation results provided in this work can be easily extended to a higher number of beams and more users to co-schedule.

Although the focus of this paper was on single-cell, the framework could be extended to case of multi-cell MU-MIMO (also known as Coordinated Multipoint (CoMP) in LTEAdvanced system) and by considering opportunistic scheduling schemes. This is left as future work.

\section{REFERENCES}

[1] C. Lim, T. Yoo, B. Clerckx, B. Lee, and B. Shim, "Recent trend of multiuser MIMO in LTE-advanced," Communications Magazine, IEEE, vol. 51, no. 3, pp. 127-135, 2013. 
[2] L. Liu, J. C. Zhang, Y. Yi, H. Li, and J. Zhang, "Combating interference: Mu-MIMO, CoMP, and HetNet (invited paper)," JCM, vol. 7, no. 9, pp. 646-655, 2012.

[3] X. Lin, J. G. Andrews, and A. Ghosh, "Modeling, analysis and design for carrier aggregation in heterogeneous cellular networks," CoRR, vol. abs/1211.4041, 2012.

[4] "3GPP TR 36.819: Technical specification group radio access network; coordinated multi-point operation for LTE physical layer aspects."

[5] Q. Spencer, C. Peel, A. Swindlehurst, and M. Haardt, "An introduction to the multi-user MIMO downlink," Communications Magazine, IEEE, vol. 42, pp. 60-67, Oct 2004.

[6] L. Liu, R. Chen, S. Geirhofer, K. Sayana, Z. Shi, and Y. Zhou, "Downlink MIMO in lte-advanced: SU-MIMO vs. MU-MIMO," Communications Magazine, IEEE, vol. 50, no. 2, pp. 140-147, 2012.

[7] A. Farajidana, W. Chen, A. Damnjanovic, T. Yoo, D. Malladi, and C. Lott, "3GPP LTE downlink system performance," in Global Telecommunications Conference, 2009. GLOBECOM 2009. IEEE, pp. 1-6, 2009.

[8] K. Kusume, G. Dietl, T. Abe, H. Taoka, and S. Nagata, "System level performance of downlink MU-MIMO transmission for 3GPP LTEAdvanced," in Vehicular Technology Conference (VTC 2010-Spring), 2010 IEEE 71st, pp. 1-5, May 2010.

[9] A. Tarighat, M. Sadek, and A. Sayed, "A multi user beamforming scheme for downlink MIMO channels based on maximizing signalto-leakage ratios," in Acoustics, Speech, and Signal Processing, 2005. Proceedings. (ICASSP '05). IEEE International Conference on, vol. 3 , pp. iii/1129-iii/1132 Vol. 3, March 2005.

[10] T. Bonald and A. Proutiere, "Wireless downlink data channels: user performance and cell dimensioning," in Proceedings of the 9th annual international conference on Mobile computing and networking (MobiCom), (New York, NY, USA), pp. 339-352, ACM, 2003.

[11] S. Sesia, I. Toufik, and M. Baker, LTE - The UMTS Long Term Evolution: From Theory to Practice. Wiley, 2011.

[12] C. B. Peel, Q. H. Spencer, A. Swindlehurst, M. Haardt, and B. M. Hochwald, Linear and Dirty-Paper Techniques for the Multiuser MIMO Downlink, pp. 209-243. John Wiley \& Sons, Ltd, 2005.

[13] Q. Spencer, A. Swindlehurst, and M. Haardt, "Zero-forcing methods for downlink spatial multiplexing in multiuser MIMO channels," Signal Processing, IEEE Transactions on, vol. 52, no. 2, pp. 461-471, 2004.

[14] C. Anton-Haro, P. Svedman, M. Bengtsson, A. Alexiou, and A. Gameiro, "Cross-layer scheduling for multi-user MIMO systems," Communications Magazine, IEEE, vol. 44, no. 9, pp. 39-45, 2006.

[15] B. Schulz, "LTE transmission modes and beamforming, white paper," tech. rep., 2011.

[16] T. Bonald, L. Massoulie, A. Proutiere, and J. Virtamo, "A queueing analysis of max-min fairness, proportional fairness and balanced fairness," Queueing Syst. Theory Appl., vol. 53, pp. 65-84, June 2006.

[17] T. Bonald and M. Feuillet, Network Performance Analysis. ISTE/Wiley, July 2011.

[18] L. Kleinrock, Queueing Systems, vol. II: Computer Applications. Wiley Interscience, 1976.

[19] G. Vivier et. al., "test scenarios and calibration cases issue 2," tech. rep.

[20] P. Kysti et. al., "Winner II channel models,tech. rep. d1.1.2 v1.2, 2007," tech. rep. 